\title{
Rotationally symmetric numerical solutions to the sine-Gordon equation
}

\author{
Olsen, O. H.; Samuelsen, Mogens Rugholm
}

Published in:

Physical Review A

Link to article, DOI:

10.1103/PhysRevA.23.3296

Publication date:

1981

Document Version

Publisher's PDF, also known as Version of record

Link back to DTU Orbit

Citation $(A P A)$ :

Olsen, O. H., \& Samuelsen, M. R. (1981). Rotationally symmetric numerical solutions to the sine-Gordon equation. Physical Review A, 23(6), 3296-3302. https://doi.org/10.1103/PhysRevA.23.3296

\section{General rights}

Copyright and moral rights for the publications made accessible in the public portal are retained by the authors and/or other copyright owners and it is a condition of accessing publications that users recognise and abide by the legal requirements associated with these rights.

- Users may download and print one copy of any publication from the public portal for the purpose of private study or research.

- You may not further distribute the material or use it for any profit-making activity or commercial gain

- You may freely distribute the URL identifying the publication in the public portal

If you believe that this document breaches copyright please contact us providing details, and we will remove access to the work immediately and investigate your claim. 


\title{
Rotationally symmetric numerical solutions to the sine-Gordon equation
}

\author{
O. H. Olsen* and M. R. Samuelsen \\ Physics Laboratory I, The Technical University of Denmark, DK-2800 Lyngby, Denmark \\ (Received 15 August 1980)
}

\begin{abstract}
We examine numerically the properties of solutions to the spherically symmetric sine-Gordon equation given an initial profile which coincides with the one-dimensional breather solution and refer to such solutions as ring waves. Expanding ring waves either exhibit a return effect or expand towards infinity. This can be explained by means of a perturbation approach. For a moderate initial radius of the shrinking ring wave we find an evolution of pulson modes. The ring waves are shown to survive the interaction between other ring waves.
\end{abstract}

\section{INTRODUCTION}

In recent years the sine-Gordon equation (SGE) has attracted considerable interest. The use of the one-dimensional SGE to model various physical phenomena, ${ }^{1}$ e.g., the propagation of fluxons on Josephson transmission lines, the propagation of crystal dislocations, and the propagation of ultrashort optical pulses is well known. The spherically-symmetric SGE has been considered as a model for nonlinear field theory as has been discussed by Derrick. ${ }^{2}$ If dissipative effects are neglected the spherically symmetric SGE is given by

$$
\phi_{r r}+[(m-1) / r] \phi_{r}-\phi_{t i}=\sin \phi .
$$

Here $m$ is the number of spatial dimensions. Equation (1.1) is a conservative, nonlinear, dispersive wave equation. For $m=1 \mathrm{Eq}$. (1.1) possesses the remarkable soliton and antisoliton solutions $(2 \pi-$ kink solutions). The one-dimensional equation also has the so-called breather solution which can be viewed as a bound state of a soliton and an antisoliton. The breather solution itself is a soliton. Unlike the $2 \pi$-kink solutions, the breather solution need not require an activation energy, because its rest energy can range from 0 to $2 E_{0}$, where $E_{0}$ is the rest energy of the kinks or antikinks. Further, breather solutions have an internal oscillatory degree of freedom which increases their physical potential.

The perturbed one-dimensional SGE, where the perturbing terms represent dissipative effects, energy input, and various kinds of impurities has been analyzed by McLaughlin and Scott $^{3}$ by means of a Greens function technique. Specific analyses of breather solutions to the perturbed SGE's can be found in papers by Scott, ${ }^{4}$ Inoue and Chung, and Inoue. ${ }^{5}$ Experiments in numero on $2 \pi-k i n k$ solutions to the one-dimensional perturbed SGE have been reported in various papers. ${ }^{6}$

No analytical solutions have been found to the three-dimensional spherically-symmetric SGE $\left[m=3\right.$ in (1.1)] but Christiansen and $\mathrm{Olsen}^{7}$ have investigated symmetric solitary wave solutions ( $2 \pi$ kinks) to Eq. (1.1) with $m=2,3$ by means of numerical computations. They referred to such solutions as ring waves and found that an expanding ring wave reaches a maximum size, depending on the initial condition, and then shrinks. This phenomenon was called a return effect and explained by Samuelsen ${ }^{8}$ who applied an energy approach. The behavior of the ring waves in the vicinity of $r=0$ has been examined by Bogolubskii and Makhankov, ${ }^{9}$ and Bogolubskii ${ }^{10}$ who found pulsating solutions. They named the solutions pulsons and studied their collapse in time. In Ref. 7 the interaction between two ring waves were investigated numerically. The ring waves were shown to behave as single waves before and after the interaction. Thus the waves were referred to as quasisolitons.

A preliminary investigation of breatherlike solutions to Eq. (1.1) with $m=3$ has been performed by Olsen and Samuelsen. ${ }^{11}$ Expanding ring waves given an initial profile which coincides with the one-dimensional breather solution were shown to either exhibit a return effect or to expand towards infinity.

In the present paper a detailed numerical investigation of breatherlike solutions to the threedimensional SGE (1.1) with $m=3$ is reported. The perturbation analysis in Ref. 3 is applied to explain the behavior of the various solutions. For moderate values of the initial radius of the ring wave we find that the wave after a period of radiation developes a new pulson mode. The light pulson mode found by Bogolubsky ${ }^{12}$ is also observed. Finally we investigate the interaction between breatherlike ring waves. The ring waves are shown to be essentially unchanged by the interaction.

The paper is structured as follows: Sec. II presents a numerical investigation of breatherlike ring waves. In Sec. III the perturbation approach is applied. The behavior of the ring waves in the 
vicinity of $r=0$ is examined in Sec. IV, while Sec. $\mathrm{V}$ contains results concerning the interaction between ring waves. Finally, in Sec. VI the results are discussed.

\section{NUMERICAL RESULTS FOR A SINGLE RING WAVE}

In this section we consider an initial-value problem for the SGE in spherical symmetry [Eq. (1.1) with $m=3$ ] imposing the initial conditions

$$
\phi(r, 0)=\psi(r, 0),
$$

and

$$
\phi_{t}(r, 0)=\psi_{t}(r, 0)
$$

where

$\psi(r, t)=4 \arctan \left(\tan \theta \frac{\sin \left[\gamma(v) \cos \theta\left(t-v r-t_{0}\right)\right]}{\cosh [\gamma(v) \sin \theta(r-R)]}\right)$,

$\gamma(v)=\left(1-v^{2}\right)^{-1 / 2}$,

$R=v t+r_{0}$,

and

$$
\tan \theta=\frac{\left(1-\omega^{2}\right)^{1 / 2}}{\omega}
$$

We have chosen the initial conditions (2.1)-(2.2) so that they coincide with the one-dimensional breather solution, in which $v$ may be conveniently interpreted as the initial velocity, $t$ being the time variable, and $t_{0}$ a phase constant. The parameter $\theta$ determines the initial amplitude and the terms $\gamma(v) \sin \theta$ and $\gamma(v) \cos \theta$ determine the initial width and frequency of oscillation, respectively. Thus we may interpret the solution of (1.1)-(2.2) as a ring wave with the initial radius $r_{0}$.

The numerical results are obtained by means of a computer program based on the method of characteristics. In Fig. 1 we display the re-

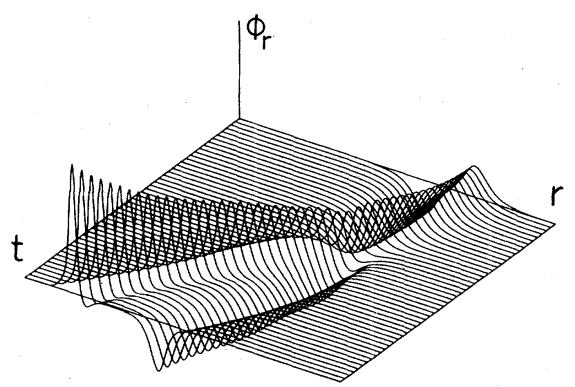

FIG. 1. The function $\phi_{r}(r, t)$ obtained by numerical solution of the initial-value problem (1.1), (2.1), and (2.2) for $m=3, r_{0}=30, \tan \theta=5$, and $v=0$. The results are displayed for $10 \leqslant r \leqslant 40$ and $0 \leqslant t \leqslant 24$. The ring wave dissociates into two ring waves.

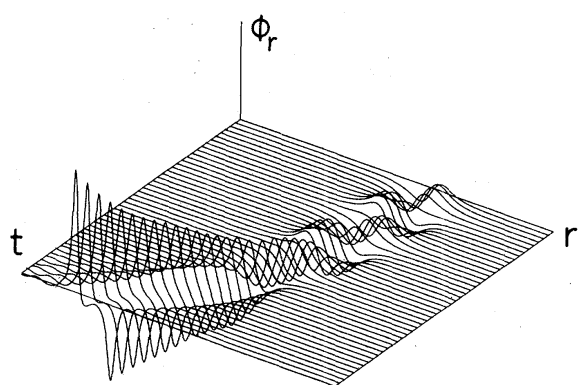

FIG. 2. The function $\phi_{r}(r, t)$ obtained by numerical solution of the initial-value problem (1.1), (2.1), and (2.2) for $m=3, r_{0}=30, \tan \theta=1$, and $v=0$. The results are displayed for $5 \leqslant r \leqslant 45$ and $0 \leqslant t \leqslant 40.5$. The ring wave oscillates several times before dissociating.

sults obtained by numerical solution of the initialvalue problem (1.1), (2.1), and (2.2) in terms of the radial derivative $\phi_{r}$ with $r_{0}=30, \tan \theta=5$, and $v=0$. Thus the ring wave is intially at rest. As time increases the ring wave shrinks. After one half-period of oscillation it dissociates into two individual ring waves, a kink from 0 to $2 \pi$ being followed by an antikink from $2 \pi$ to 0 .

Next, we have solved (1.1), (2.1), and (2.2) with $r_{0}=30, \tan \theta=1$, and $v=0$. Also in this case the ring wave initially is at rest, but compared to the first example the energy is smaller (see the next sections). In Fig. 2 the results are again displayed in terms of $\phi_{r}$. The ring wave now oscillates several times over a longer period of time before its starts shrinking and finally splits into two individual ring waves.

Figure 3 shows the results when we choose $r_{0}$ $=30, \tan \theta=0.4$, and $v=0$ in (1.1), (2.1), and (2.2). These parameter values correspond to a smaller initial energy than in the former examples. The ring wave, initially at rest, oscillates for a long time but starts shrinking and will eventually dis-

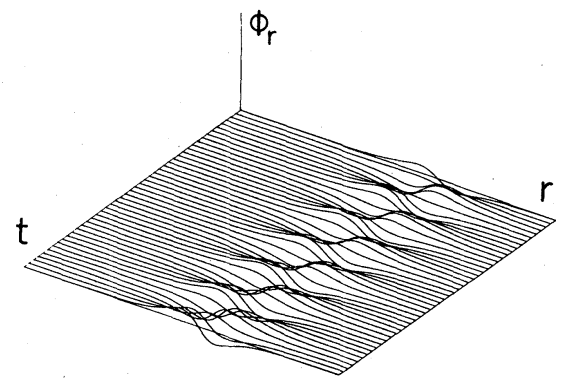

FIG. 3. The function $\phi_{r}(r, t)$ obtained by numerical solution of the initial-value problem (1.1), (2.1), and (2.2) for $m=3, r_{0}=30, \tan \theta=0.4$, and $v=0$. The results are displayed for $5 \leqslant r \leqslant 45$ and $0 \leqslant t \leqslant 40.5$. The ring wave oscillates and will eventually shrink. 
sociate into two individual ring waves.

The behavior of the ring waves with zero initial velocity is explained in the next section by means of a perturbation approach.

Now, we consider ring waves given an initial outward velocity. In Fig. 4 we show the solution of (1.1), (2.1), and (2.2) in terms of $\phi_{r}$. The parameter values are $r_{0}=30, \tan \theta=5$, and $v=0.5$, corresponding to Fig. 1 but with a nonzero initial velocity. At early times the oscillating ring wave expands but the radial velocity decreases such that a maximum radius for the ring wave is reached and the ring wave exhibits a return effect. Then the ring wave starts shrinking towards the center in the manner shown in the previous figures.

In Fig. 5 the parameter values in (1.1), (2.1), and (2.2) are $r_{0}=30, \tan \theta=1$, and $v=0.5$. In this case we find that the outward ring wave expands towards infinity at a constant velocity. The frequency of oscillation increases towards unity.

Finally in this section we show another example of an expanding ring wave. In Fig. 6 we have chosen the parameter values in (1.1), (2.1), and (2.2) to be $r_{0}=15, \tan \theta=1$, and $v=0.8$. We observe the same qualitative behavior as observed in Fig. 5. The expansion of ring waves towards infinity can also be explained by the perturbation approach given in the next section.

\section{PERTURBATION ANALYSIS}

The qualitative behavior of the numerical solutions to the SGE obtained in the previous section can be understood by application of the perturbation method of McLaughlin and Scott, ${ }^{3}$ and Scott. ${ }^{4}$ The idea is to consider the term $[(m-1) / r] \phi_{r}$ in Eq. (1.1) as a perturbation of the one-dimensional SGE. The expression Eq. (2.2a) is an exact solution of the one-dimensional SGE with the energy ${ }^{4}$

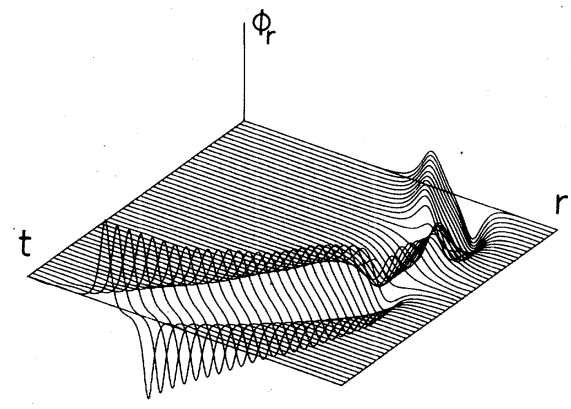

FIG. 4. The function $\phi_{r}(r, t)$ obtained by numerical solution of the initial-value problem (1.1), (2.1), and (2.2) for $m=3, r_{0}=30, \tan \theta=5$, and $v=0.5$. The results are displayed for $10 \leqslant r \leqslant 40$ and $0 \leqslant t \leqslant 40$. The ring wave exhibits a return effect and dissociates.

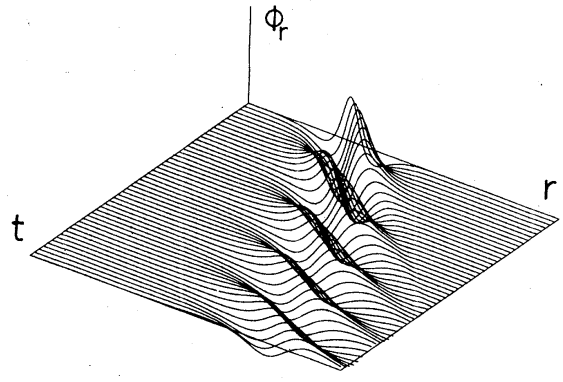

FIG. 5. The function $\phi_{r}(r, t)$ obtained by numerical solution of the initial-value problem (1.1), (2.1), and (2.2) for $m=3, r_{0}=30, \tan \theta=1$, and $v=0.5$. The results are displayed for $20 \leqslant r \leqslant 60$ and $0 \leqslant t \leqslant 35$. The ring wave expands towards infinity.

$$
\boldsymbol{H}=16\left(1-\omega^{2}\right)^{1 / 2} \gamma(v) \quad(v=\stackrel{\circ}{R}),
$$

and momentum ${ }^{4}$

$$
P=16\left(1-\omega^{2}\right)^{1 / 2} v \gamma(v),
$$

where $\omega, v$, and $R$ now are the instantaneous frequency parameter, velocity, and position (radius) of the breather solution. From Ref. 4 it then follows that the time evolution of $H$ and $P$ due to the perturbing term $[(m-1) / r] \phi_{r}$ is given by

$$
\begin{aligned}
\dot{H} & =(m-1) \int \frac{\phi_{r} \phi_{t}}{r} d r \\
& \simeq \frac{m-1}{R} \int \phi_{r} \phi_{t} d r=-\frac{m-1}{R} P,
\end{aligned}
$$

and

$$
\begin{aligned}
\dot{P} & =-(m-1) \int \frac{\phi_{r}^{2}}{r} d r \\
& \simeq-\frac{(m-1)}{R} \int \phi_{r}^{2} d r,
\end{aligned}
$$

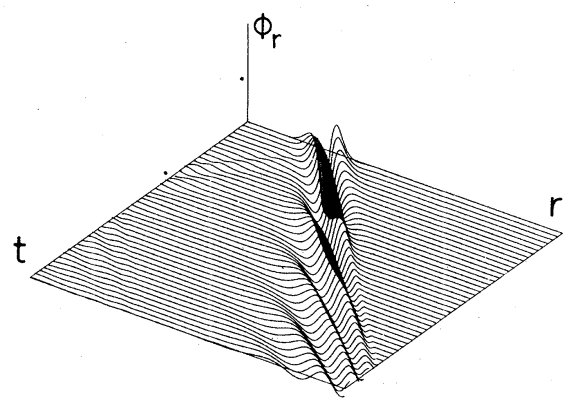

FIG. 6. The function $\phi_{r}(r, t)$ obtained by numerical solution of the initial-value problem (1.1), (2.1), and (2.2) for $m=3, r_{0}=15, \tan \theta=1$, and $v=0.8$. The results are displayed for $5 \leqslant r \leqslant 45$ and $0 \leqslant t \leqslant 30$. The ring wave expands towards infinity. 
both valid for $R \gg 1$. Thus (3.3), (3.1), and (3.2) $(P=v H)$ yields

$$
H R^{m-1}=\text { constant . }
$$

The simple energy consideration in Ref. 8 gives the same result. On the other hand the perturbation method used here will for a single soliton with the appropriate expressions for the energy $H$ and momentum $P$, yield exactly the results of Ref. 8 giving good confidence in the method.

In order to determine the time evolution of $R$ and $\omega$ it is necessary to calculate the integral in Eq. (3.4). This has not been performed but generally it will be a function of $t_{0}, R, v, \omega$ and periodic in time with the frequency $2 \omega / 2 \pi \gamma(v)$. In two special cases, however, the integral has been calculated. For $\omega \rightarrow 1$ and $v \gg\left(1-\omega^{2}\right)^{1 / 2}$, corresponding to a very tight binding of the two solitons, the result is

$$
\int \phi_{r}^{2} d r=16\left(1-\omega^{2}\right)^{1 / 2} v^{2} \gamma(v)=v P .
$$

From $P=v H$ we get

$$
\frac{\dot{v}}{v}=\frac{\dot{P}}{P}-\frac{\dot{H}}{H} .
$$

Inserting Eq. (3.3) and Eq. (3.4) with Eq. (3.6) into Eq. (3.7) yields

$$
\dot{v}=0 \text {. }
$$

Thus the velocity $v$ is a constant. This corresponds to the behavior seen in Figs. 5 and 6 .

The second case is for $v=0$. Then the integral becomes

$$
\int \phi_{r}^{2} d r=16\left(1-\omega^{2}\right)^{1 / 2}\left(1-\frac{2 Z}{\sinh 2 Z}\right),
$$

where

$$
Z=\sinh ^{-1}\left(\frac{\left(1-\omega^{2}\right)^{1 / 2}}{\omega} \sin \omega\left(t-t_{0}\right)\right)
$$

This result can be used to calculate $\dot{v}$ and $\dot{\omega}$ for small velocities. From Eq. (3.7) it follows that

$$
\dot{v}=-\frac{m-1}{R}\left(1-\frac{2 Z}{\sinh 2 Z}\right) \text {, }
$$

while Eq. (3.1) yields

$$
\dot{\omega}=v \frac{(m-1)}{R} \frac{\left(1-\omega^{2}\right)}{\omega} \frac{2 Z}{\sinh 2 Z} .
$$

For smaller values of $Z$, i.e., smaller values of $\tan \theta[\omega \rightarrow 1$, see Eq. (2.2) $] 2 Z / \sinh 2 Z$ will be closer to but still smaller than one. Therefore from (3.11) and (3.12) it follows that the changes in velocity and frequency will be slower for smaller values of $\tan \theta$. This behavior can be seen in Figs. 1, 2, and 3 where the value of $\tan \theta$ is decreasing with increasing figure number corre- sponding to increasing values of $\omega$. For smaller values of $\tan \theta$ the ring wave oscillates several times and for a longer time before it starts shrinking and finally splits into individual ring waves.

One can get a more quantitative check of the description of this behavior by expanding Eq. (3.11). If $\tan \theta=\left(1-\omega^{2}\right)^{1 / 2} / \omega \ll 1 \mathrm{Eq}$. (3.11) becomes

$$
\dot{v}=-\frac{m-1}{R} \frac{2}{3} \tan ^{2} \theta \sin ^{2}\left[\omega\left(t-t_{0}\right)\right] .
$$

As long as $v$ is small it follows from Eq. (3.12) that $\omega$ remains constant. For large $t$ only the mean value of the squared sine will contribute to Eq. (3.13). Therefore the deviation of $r$ from $r_{0}$, $\Delta \boldsymbol{r}$ is given by

$$
\Delta r=-\frac{m-1}{R} \frac{\tan ^{2} \theta}{6} t^{2}
$$

We have compared the value of $-\Delta r / t^{2}$ calculated from Eq. (3.14) with the value determined from Figs. 1, 2, and 3 by calculating the ratios between the two values obtained. For $\tan \theta=0.4$ (Fig. 3) we find 1.02, for $\tan \theta=1$ (Fig. 2) 1.23, and for $\tan \theta=5$ (Fig. 1) 11.3. It is seen that the agreement is good for small $\tan \theta$ values.

It is difficult to use the perturbation analysis described here except for the two special cases we have considered $(\omega \simeq 1$ and $v \simeq 0)$. This is first due to the complicated nature of the integral itself and second to the fact that the parameters that determine the breather solution (especially $\omega$ ) in most cases vary rapidly, even within a single period. Another difficulty is that to the extent the initial phase constant $t_{0}$ enters the results, the time variation of $t_{0}$ should be included.

In this section we have (to some extent) explained the two different kinds of solutions, the ever expanding ring wave and the returning ring wave. Finally we remark that besides the initial radius $r_{0}$, velocity $v$, and frequency parameter $\tan \theta$ the resulting ring wave depends on the initial phase constant $t_{0}$. For instance the two sets of initial values, both for $r_{0}=30, v=0.5, \tan \theta=1$, and $v=0.7, \tan \theta=5$ yield returning ring waves or even expanding ring waves depending on the value of $t_{0}$.

\section{EVOLUTION OF RING WAVES INTO PULSON MODES}

In order to study the behavior of the ring waves in the vicinity of $r=0$ we choose the condition

$$
\phi_{r}(0, t)=0 \text {. }
$$

The presence of the factor $(m-1) / r$ in Eq. (1.1) forces the condition (4.1) to be fulfilled if $\phi$ is to be regular. Insertion of a power series expansion of $\phi$ in $r$ into (1.1) shows this. Further- 


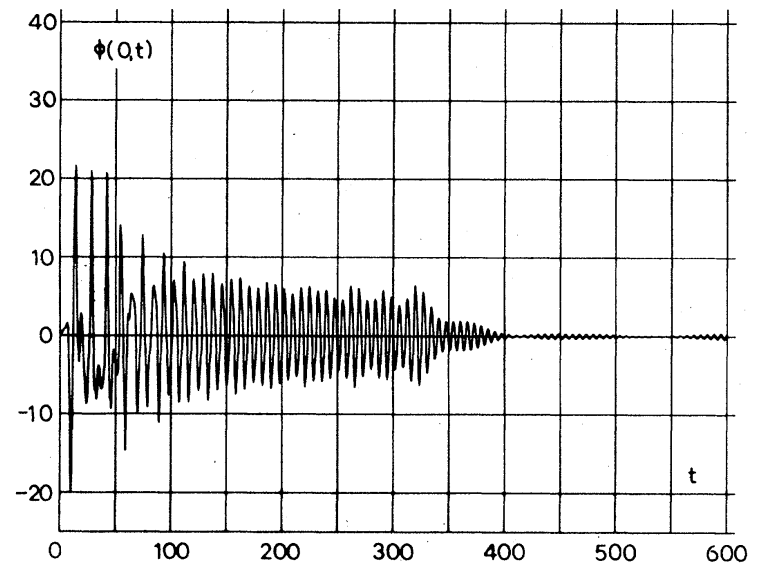

FIG. 7. The function $\phi(0, t)$ obtained by numerical solution of the initial-value problem (1.1), (2.1), and (2.2) with the condition (4.1) for $m=3, v=0, \tan \theta=1, r_{0}=6$ and $t_{0}=0$. At $t \simeq 100, \phi(0, t)$ enters a light pulson mode, oscillating around 0 . The pulson collapses at $t \simeq 350$.

more, this condition prevents the point $r=0$ from being either a source or a drain. This can be seen by calculating the energy flux, $Q$, through a sphere of radius $r$ :

$$
Q(t)=-4 \pi r^{2} \phi_{r} \phi_{t} .
$$

We solve the initial-value problem (1.1), (2.1), and (2.2) with the condition (4.1) where $v=0, \tan \theta$ $=1, r_{0}=6$, and $t_{0}=0$ [in order to satisfy (4.1) for $t=0] .{ }^{13}$ In Fig. 7 the results are displayed in terms of $\phi(0, t)$. For $t=80$ we find that approximately half of the energy

$$
E=\int_{0}^{r_{\max }}\left[\frac{1}{2} \phi_{r}^{2}+\frac{1}{2} \phi_{t}^{2}+(1-\cos \phi)\right] 4 \pi r^{2} d r
$$

of the initial ring wave is radiated towards infinity. ${ }^{14}$ The value of $r_{\max }$ in Eq. (4.3) is set equal to 24. At $t \simeq 100$ a light pulson mode is formed. The pulson mode is modulated and slightly radiating. At $t \simeq 350$ the pulson is destroyed because of the radiation and the rest of the energy is radiated towards infinity. Similar results have been found for other parameter values (e.g., $v=0, \tan \theta=1$, $r_{0}=8,10,12$, and $\left.t_{0}=0\right)$. The same qualitative behavior has been observed when the initial conditions are chosen as a kink from zero in the vicinity of $r=0$ to $2 \pi$ for $r \gg 1 .^{9}$

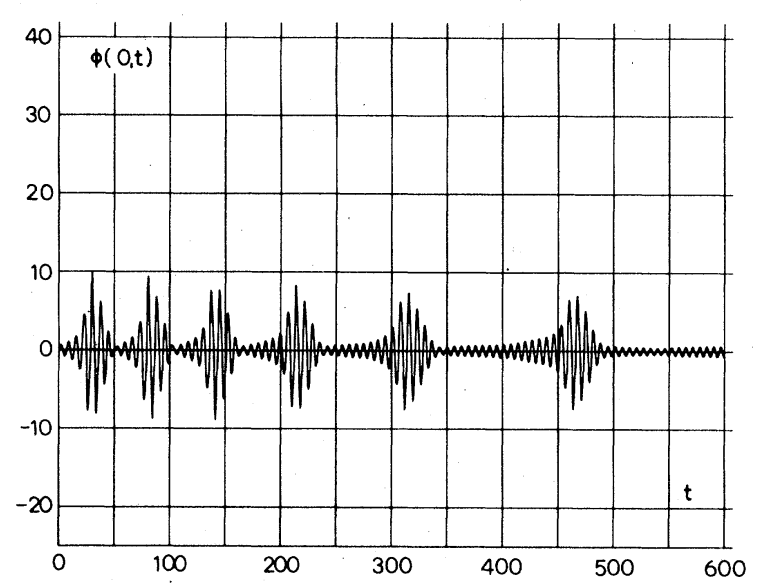

FIG. 8. The function $\phi(0, t)$ obtained by numerical solution of the initial-value problem (1.1), (2.1), and (2.2) with the condition (4.1) for $m=3, v=0, \tan \theta=0.2$, $r_{0}=6$, and $t_{0}=0$. A modulated mode is observed.

Next we solve the initial-value problem (1.1), (2.1), and (2.2) with the condition (4.1) where $v=0$, $\tan \theta=0.2, r_{0}=6$, and $t_{0}=0$.

The results are shown in Fig. 8 and are again displayed in terms of $\phi(0, t)$. The initial energy in this case is less than half of the initial energy of the former case. Immediately a modulated mode is formed. At $t=300$ only one third of the initial energy is radiated towards infinity. The energy is slowly radiated while the modulated wave expands, reaches a maximum extent and then returns. Finally the wave reaches the linear limit and propagates towards infinity. For other initialvalue problems we find similar results, e.g., for $v=0, \tan \theta=0.25(0.5), r_{0}=6$, and $t_{0}=0$. An explanation of why we find the evolution of different modes is found in the fact that for small initial energies no dissociation into a kink and an antikink takes place before the collapse at $r=0$.

We have not been able to explain these phenomena by any analytic approximations. The method applied by Bogolubsky ${ }^{10}$ for the $\phi^{4}$ model does not work because the amplitudes are too large.

Whether such oscillating solutions are of interest as models of mesons is still an open question.

\section{INTERACTION BETWEEN RING WAVES}

The question of stability of the ring waves for $r \gg 1$ is examined in this section by means of numerical computations. We solve the initial-value problem (1.1) and (2.1) with

$$
\psi(r, t)=4 \sum_{i=1}^{2} \arctan \left(\tan \theta_{i} \frac{\sin \left[\gamma\left(v_{i}\right) \cos \theta_{i}\left(t-v_{i} r-t_{0 i}\right)\right]}{\cosh \left[\gamma\left(v_{i}\right) \sin \theta_{i}\left(r-R_{i}\right)\right]}\right)
$$




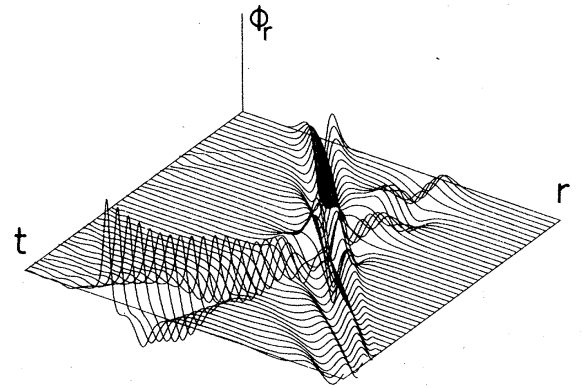

FIG. 9. The function $\phi_{r}(r, t)$ obtained by numerical solution of the initial-value problem (1.1), (2.1), and (6.1) for $m=3, r_{01}=15, \tan \theta=1, v_{1}=0.8, r_{02}=30$, $\tan \theta_{2}=1$, and $v_{2}=0$. Collision of expanding and shrinking ring wave. The ring waves survive the collision.

where

$$
\begin{aligned}
& \gamma\left(v_{i}\right)=\left(1-v_{i}^{2}\right)^{-1 / 2}, \\
& R_{i}=v_{i} t+r_{0 i},
\end{aligned}
$$

and

$$
\tan \theta_{i}=\frac{\left(1-\omega_{i}\right)^{1 / 2}}{\omega_{i}}
$$

Initially, we have two ring waves, separated for $r_{02} \gg r_{01} \gg 1$, which coincide with one-dimensional breather solutions. The parameter $v_{i}$ may be interpreted as the initial velocity while the parameter $\theta_{i}$ determines the initial amplitude, width, and frequency of oscillation for the $i$ th ring wave.

In Fig. 9 the interaction between the ring waves shown in Figs. 6 and 2 is displayed again in terms of $\phi_{r}$. Thus the parameter values in (5.1) are $r_{01}=15, \tan \theta_{1}=1, v_{1}=0.8, r_{02}=30, \tan \theta_{2}=1$, and $v_{2}=0$. It is seen that the two waves retain their identity after the collision. The outward wave continues to expand while the other wave oscillates before it shrinks. Both waves develop as single waves. As a result of the collision we observe small phase jumps in the propagation directions.

A second numerical experiment is shown in Fig. 10. The ring wave from Fig. 6 interacts with the ring wave from Fig. 3. The parameter values in (5.1) are $r_{01}=15, \tan \theta_{1}=1, v_{1}=0.8, r_{02}=30$, $\tan \theta=0.4$, and $v_{2}=0$. Also in this case the colliding ring waves survive the interaction. The

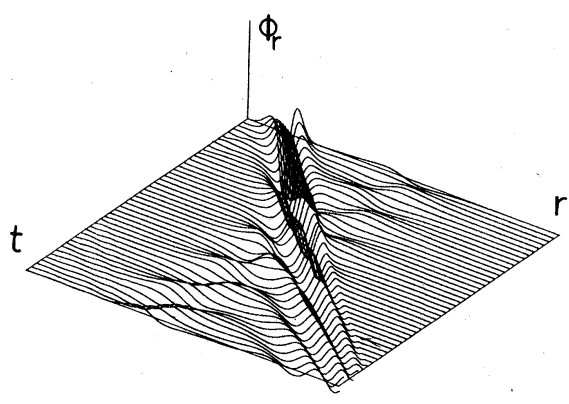

FIG. 10. The function $\phi_{r}(r, t)$ obtained by numerical solution of the initial-value problem (1.1), (2.1), and (6.1) for $m=3, r_{01}=15, \tan \theta_{1}=1, v_{1}=0.8, r_{02}=30$, $\tan \theta_{2}=0.4$, and $v_{2}=0$. Collision of expanding and shrinking ring wave. The ring waves survive the collision.

contracting ring wave eventually dissociates. Again small phase jumps in the propagation directions are observed.

\section{DISCUSSION}

In this paper we have examined ring-wave solutions to the sine-Gordon equation in three dimensions by means of numerical computations. Ring waves with an initial profile which coincides with the one-dimensional breather solution exhibit either a return effect, eventually dissociating into individual ring waves, or expand towards infinity. Similar results have been found in two dimensions. The dissociation is due to the fact that the energy per unit area increases during the shrinking process. The behavior of the ring waves expanding towards infinity and the ring waves with zero initial velocity can be well understood qualitatively by a simple perturbation approach. We have not been able to treat these highly nonlinear problems quantitatively.

In the vicinity of $r=0$ we find that the ring waves collapse into pulson modes. The light pulson mode discovered by Bogolubsky is found. Furthermore, we have observed a new modulated mode. Whether these modes are of any physical importance is an open question.

The ring waves are shown to preserve their identity after a mutual collision. This indicates the minor importance of the term $[(m-1) / r] \phi_{r}$ for $r \gg 1$. In this limit the problem is essentially one dimensional.
* Present address: NIRO Atomizer, R \& D Systems and Design Division 305, Gladsaxevej, DK-2860 Soeborg, Denmark.

${ }^{1}$ A. Barone, F. Esposito, C. J. Magee, and A. C. Scott,
Riv. Nuovo Cimento 1, 227 (1971).

${ }^{2}$ G. H. Derrick, J. Math. Phys. (N.Y.) 5, 1252 (1964). ${ }^{3}$ D. W. McLaughlin and A. C. Scott, Phys. Rev. A 18 , 1652 (1978). 
${ }^{4}$ A. C. Scott, Phys. Scr. 20, 509 (1979).

${ }^{5}$ M. Inoue and S. G. Chung, J. Phys. Soc. Jpn. 46, 1594 (1979); M. Inoue, ibid. 47, 1723 (1979).

${ }^{6}$ See, e.g., K. Nakajima, $\bar{Y}$. Onodera, and Y. Ogawa, J. Appl. Phys. 47, 1620 (1976); or P. L. Christiansen and O. H. Olsen, Wave Motion 2, 185 (1980).

${ }^{7}$ P. L. Christiansen and O. H. Olsen, Phys. Scr. 20 , 531 (1979).

${ }^{8}$ M. R. Samuelsen, Phys. Lett. 74A, 21 (1979).

${ }^{9}$ I. L. Bogolubskii and V. G. Makhanov, Zh. Eksp. Teor. Fiz. Pis'ma Red 24, 15 (1976) [JETP Lett. 24, 12 (1976)].
${ }^{10}$ I. L. Bogolubskii, Zh. Eksp. Teor. Fiz. Pis'ma Red. 24, 579 (1976) [JETP Lett. 24, 535 (1976)].

${ }^{11} \mathrm{O}$. H. Olsen and M. R. Samuelsen, Phys. Lett. $\underline{77 \mathrm{~A}}$, 95 (1980).

${ }^{12}$ I. L. Bogolubsky, Phys. Lett. 61A, 205 (1977).

${ }^{13}$ For $r=0$ we write Eq. (1.1) with $m=3$, applying the condition Eq. (4.1), $3 \phi_{r r}-\phi_{t t}=\sin \phi$. The solution at $r=0$ is determined by solving this equation.

${ }^{14}$ In the numerical calculation we have chosen a condition for large $r$ which absorbs the radiation, i.e., the right side of Eq. (1.1) is replaced by $\sin \phi+0.025$ $(r-54)^{3} \phi_{t}$ for $r \geqslant 54$. 